\title{
Adaptation of The N170-Component of the Event- Related Brain Potential to Single Letters and Words in Adults and Children
}

\author{
Ulrike Kagel \\ University of Bamberg \\ Jascha Rüsseler ( $\nabla$ jascha.ruesseler@uni-bamberg.de ) \\ University of Bamberg
}

\section{Research Article}

Keywords: reading, N170, adaptation, words, letters, event-related brain potentials.

Posted Date: January 27th, 2021

DOl: https://doi.org/10.21203/rs.3.rs-150537/v1

License: (c) (1) This work is licensed under a Creative Commons Attribution 4.0 International License.

Read Full License 


\title{
Adaptation of the N170-component of the event-related brain potential to single letters and words in adults and children
}

\author{
Ulrike Kagel $^{1,2}$, Jascha Rüsseler ${ }^{1,2 *}$ \\ ${ }^{1}$ Department of Psychology, University of Bamberg, Bamberg, Germany \\ ${ }^{2}$ Bamberg Graduate School of Affective and Cognitive Sciences (BAGrACS), University of \\ Bamberg, Bamberg, Germany \\ * Correspondence: \\ jascha.ruesseler@uni-bamberg.de
}

Keywords: reading, N170, adaptation, words, letters, event-related brain potentials.

\begin{abstract}
Background. Neural specialization for print develops during learning to read and can be studied with event-related brain potentials (ERPs). Neural adaptation refers to smaller neural responses when a stimulus or a class of stimuli is repeated several times. Previously, neural specialization for print processing in adults has been shown to be reflected in the word-specific N170 component of the ERP. Furthermore, adapation of the N170 elicited by words has been found to correlate with reading speed. Here, we investigated adapation of the N170-component evoked by single letters, words and faces in seven to nine- year old German-language speaking children and in a group of adult normal readers.

Results. For adults, we replicated previous reports of adaptation of N170-amplitude at posterior-temporal electrodes P7 and P8 for single letters and words. For children, we only observed adaptation of N170-amplitude for single letters. No adaptation of N170-amplitude was apparent for words. However, in both, children as well as adults, N170-adaptation did not correlate with measures of reading speed. For faces, that served as a non-linguistic control condition, no adaptation of N170 amplitude was observed.
\end{abstract}

Conclusions. We conclude that adaptation of the word-specific N170 component of the ERP might be linked to increasing perceptual experience with letters and words. 


\section{Background}

Temporal dynamics of the neural specialization for print can be studied with methods that have a high temporal resolution like event-related brain potentials (ERPs) or magnetencephalography / magnetic source imaging (MEG/MSI). The N170 component of the ERP peaks approximately $140 \mathrm{~ms}$ to $200 \mathrm{~ms}$ after stimulus presentation (e.g. 1, 2) at posteriortemporal electrode sites and strongly depends on the perceptual familiarity of certain stimulus classes. Printed words or single letters of a known script are processed differently when compared to various control stimuli like faces, shapes, or false font strings starting approximately 150ms after stimulus presentation (3,4). Larger N170-components have been consistently reported for word-like stimuli and single letters compared to a range of visual control stimuli in fluent readers (for single letters: 5, 6; for words: e.g. 3, 7-13). In a longitudinal study, Maurer and colleagues showed that in pre-literate children, N170 amplitude did not differ between words and symbol strings (11), but was higher for words compared to symbols after the first two years of formal reading education (12). Furthermore, it was shown that N170 specialization for print is largely absent in adults who cannot read and write properly (14).

Neural adaptation or habituation refers to smaller neural responses when a stimulus or a class of stimuli (e.g. faces) is repeated (15). It has been shown that repeated presentation of words (the same word or different words) leads to a reduction in N170- amplitude, indicating neuronal response specificity for words and faces, respectively, as a stimulus category $(5,16)$. Interestingly, Korinth et al. (5), studying young adults (mean age ca. 22 yrs.), observed an adaptation effect of a subcomponent of the N170 amplitude for fast, but not for slow readers. This might reflect different degrees of neuronal response specificity in fast and slow readers. Fu et al. (17) and Cao et al., (16,18) observed N170 adaptation for Chinese characters for short inter-stimulus-intervals (ISIs), but Maurer et al. (9) and Mercure et al. (19) did not observe N170 adaptation for words with longer ISIs of $1000 \mathrm{~ms}$ or $1500 \mathrm{~ms}$. However, it is unclear whether the difference between Chinese characters and words written in an alphabetic script contributes to the observed differences.

Cao et al. (16) further showed that the word-related adaptation effect is larger when the same word is repeated compared with a condition where different words are presented in succession. Again, Chinese characters and a short ISI of 200ms were used. In the present study, we used a variable ISI (600ms to $1200 \mathrm{~ms}$ ) and the same stimulus as an adaptor to study the habituation of the N170. Furthermore, we investigated whether the N170 habituation is already present in eight-year old children. 
Another important finding with respect to the N170 is its laterality: it has been consistently reported that N170-amplitude for words, letters, and pseudowords is larger over left-hemisphere regions. This is the case for absolute N170-amplitude as well as for the difference between word-like and low-level visual control stimuli $(3,9,11,20 ; 21$; for a similar result using MEG/MSI, see 22). However, beginning readers (children) are less likely to display a left lateralized N170 to word-like stimuli (23) and the same holds true for adults that cannot read properly (14). Thus, it seems that the relative left-lateralization for words does not show up until words are automatically mapped to left-lateralized phonological processing systems of the brain. This so-called phonological mapping hypothesis states that relative N170lateralization emerges whenever automatic links between orthography and phonology are activated during the processing of word-like stimuli $(24,25)$. The small set of studies of single letter processing in monolingual speakers shows inconsistent results with respect to N170 leftlateralization: Tarkiainen et al. (22), Park et al. (26) and Stevens et al. (6) reported larger N170 amplitudes to letters relative to control stimuli primarily for left-hemisphere regions, whereas Wong et al. (27) found a difference between letters and control stimuli in early occipitotemporal processing in both hemispheres. Moreover, Pernet et al. (28) reported no amplitude differences between letters, geometrical figures, faces, tools, structured textures and Asiatic characters in the first $220 \mathrm{~ms}$ of processing.

In the present study, we extend earlier results by showing adaptation of the N170 to single letters, but not words or faces in a group of eight-year old typical readers and, for single letters and words, in a group of reading-proficient adults. Furthermore, we set out to assess possible differences in N170- adaptation between the left and the right hemisphere of the brain. We expected differences in neural adaptation and in lateralization of the N170 and the N170adaptation effect between children and adult readers, i.e. readers with different reading expertise. More specifically, we expected stronger adaptation and larger left-lateralization of the N170 in faster readers, i.e. in adults compared to children.

\section{Results}

Reading tests

Adults. Saarbruecker Lesetexte. On average, adults needed 321s to read the six short stories (range: $236 \mathrm{~s}$ to $397 \mathrm{~s} ; \mathrm{SD}=47 \mathrm{~s}$ ). Error rate was low and is not analyzed. Normative 
values are given for reference purposes: $382 \mathrm{~s}, \mathrm{SD}=63.44 \mathrm{~s} ; \mathrm{n}=60$ adults between 17 and 67 yrs. Thus, the present sample can be characterized as a sample of fast readers.

Salzburger Lesetexte. Adults read 59.6 sentences in three minutes correctly (range: 45 to $91 ; \mathrm{SD}=12$ ).

Children. SLRT-II. Children read 61,67 words (SD = 18,35, range 22 to 90) and 36,05 pseudowords ( $\mathrm{SD}=10.83$, range: 11 to 51 ) correctly.

Behavioural measures. Response times and accuracy

Adults. Response times differed between the three conditions (letters: $M=411 \mathrm{~ms}$, SD $=41 \mathrm{~ms}$, words: $\mathrm{M}=430 \mathrm{~ms}, \mathrm{SD}=44 \mathrm{~ms}$; faces: $\mathrm{M}=470 \mathrm{~ms}, \mathrm{SD}=47 \mathrm{~ms}$. A within groups ANOVA with factors CONDITION (3; single letters, words, faces) x BLOCK (3; A, B and C) revealed a reliable effect of CONDITION $\left(\mathrm{F}_{(2,42)}=29.99, \mathrm{p}=.0001\right)$ but no significant main effect of or interaction with the BLOCK-factor (p's > .17). Simple contrasts indicate that response times of all three conditions differed reliably from each other (all p's $<.01$ ).

Mean response accuracy was very high for all conditions: letters $(\mathrm{M}=99.8 \%, \mathrm{SD}=$ $0.01)$, words $(\mathrm{M}=99.5 \%, \mathrm{SD}=0.017)$ and faces $(\mathrm{M}=99.2 \%, \mathrm{SD}=.033)$ with no significant main effects or interaction in a CONDITION (3) by BLOCK (3) ANOVA.

Due to the low number of false alarms (mean over all conditions and blocks $=0.86$ per participant) these are not analyzed any further.

Children. Mean response times (letters: $\mathrm{M}=505 \mathrm{~ms}, \mathrm{SD}=57 \mathrm{~ms}$; words: $\mathrm{M}=548 \mathrm{~ms}$, $\mathrm{SD}=60 \mathrm{~ms}$; faces: $\mathrm{M}=534 \mathrm{~ms}, \mathrm{SD}=52 \mathrm{~ms}$ ) differed between the conditions (main effect of CONDITION: $\mathrm{F}_{(2,42)}=8.67, \mathrm{p}=.001$; no other significant effects). Simple contrasts indicate that RT to letters was faster compared to words $(\mathrm{p}<.01)$. All other contrasts missed significance.

Mean error rate differed between conditions (letters: $\mathrm{M}=12.6 \%, \mathrm{SD}=3.7 \%$; words: $\mathrm{M}$ $=4.7 \%, \mathrm{SD}=10.4 \%$; faces: $\left.\mathrm{M}=11.1 \%, \mathrm{SD}=3.6 \% ; \mathrm{F}_{(2,40)}=4.69, \mathrm{p}<.028\right)$. Neither the main effect BLOCK nor the two-way interaction turned out to be significant (p's > .67). Simple contrasts indicate that error rate for letters and faces is higher than for words (p's <.027).

False alarms were low and did not differ between conditions (letters: $\mathrm{M}=1.46 \%, \mathrm{SD}=$ 0.017 ; words: $\mathrm{M}=2.58 \%$; $\mathrm{SD}=0.019$; faces: $\mathrm{M}=2.54 \%, \mathrm{SD}=0.014)$. Taken together, children responded slower and made more errors compared to adults. 
ERPs

ERPs for both, adults and children, show a clear negative peak at temporo-parietal electrodes approximately 200ms post stimulus presentation (N170) followed by a broadly distributed positivity between 300ms and 600ms (see Figures 1 and 2). N170 amplitude varies with age and as a function of condition, block and electrode. This is reflected in a significant four-way interaction for N170 peak amplitude (GROUP (2) by CONDITION (3) by BLOCK (3) by LATERALITY $\left.(2): \mathrm{F}_{(4,160)}=3.69, \mathrm{p}=.044\right)$. Main effects of $\operatorname{BLOCK}\left(\mathrm{F}_{(2,80)}=5.44, \mathrm{p}\right.$ $=.006)$ and CONDITION $\left(\mathrm{F}_{(2,80)}=34,96, \mathrm{p}=.001\right)$ as well as interactions of GROUP by CONDITION $\left(\mathrm{F}_{(2,80)}=11,8, \mathrm{p}=.001\right)$, CONDITION by LATERALITY $\left(\mathrm{F}_{(2,80)}=3.98, \mathrm{p}=\right.$ $.028)$, GROUP by CONDITION by LATERALITY $\left(\mathrm{F}_{(2,80)}=6.55, \mathrm{p}=.004\right)$ also turned out to be significant. To disentangle the four-way interaction, two three-way within group ANOVAs with factors CONDITION, BLOCK and LATERALITY were computed for children and adults, respectively.

For adults, main effects of CONDITION $\left(\mathrm{F}_{(2,40)}=7.03, \mathrm{p}=.004\right)$ and $\operatorname{BLOCK}\left(\mathrm{F}_{(2,40)}\right.$ $=10.83, \mathrm{p}=.001)$ were observed. The interaction of CONDITION by BLOCK $\left(\mathrm{F}_{(4,80)}=2.03\right.$, $\mathrm{p}=$.099) just missed significance. However, one-way ANOVAs for each condition at electrodes P7 and P8 showed significant main effects of BLOCK for single letters (P7, block 1: $-5.3 \mu \mathrm{V}$, block 2: $-3.6 \mu \mathrm{V}$, block 3: $-3 \mu \mathrm{V}, \mathrm{F}_{(2,40)}=7.27, \mathrm{p}=.002$; P8, block 1: $-5.3 \mu \mathrm{V}$, block 2: $-3.6 \mu \mathrm{V}$, block 3: $-2.9 \mu \mathrm{V}, \mathrm{F}_{(2,40)}=7.98, \mathrm{p}=.002$ ) and for words at P8 (block 1: $-4.85 \mu \mathrm{V}$, block 2: $3.24 \mu \mathrm{V}$, block 3: $\left.-3.97 \mu \mathrm{V}, \mathrm{F}_{(2,40)}=3.39, \mathrm{p}=.045\right)$, but not for faces $(\mathrm{P} 7: \mathrm{p}=.159 ; \mathrm{P} 8: \mathrm{p}=$ .848 ). For single letters as well as words, N170 amplitude decreased reliably from block 1 to block 2 (single letters, P7: $F_{(1,20)}=13.24, p=.002 ; P 8: F_{(1,20)}=5.18, p=.034$; words, P8: $F_{(1}$, 20) $=9.7, \mathrm{p}=.005$ ) but not from block 2 to block 3 (single letters, $\mathrm{P} 7: \mathrm{F}_{(1,20)}=1.007, \mathrm{p}=.328$; P8: $\mathrm{F}_{(1,20)}=1.7, \mathrm{p}=.208$; words, $\left.\mathrm{P} 8: \mathrm{F}_{(1,20)}=1.38, \mathrm{p}=.253\right)$. Thus, $\mathrm{N} 170$ amplitude for adults shows habituation for single letters and for words, but not for faces at posterior-temporal electrodes (see Figure 3 for the topography of the adaptation effect).

For children, the main effect of CONDITION $\left(\mathrm{F}_{(2,40)}=30.55, \mathrm{p}=.001\right)$, the two-way interaction CONDITION by LATERALITY $\left(\mathrm{F}_{(2,40)}=9.7, \mathrm{p}=.001\right)$ and the three-way interaction CONDITION by BLOCK by LATERALITY $\left(\mathrm{F}_{(4,80)}=2.75, \mathrm{p}=.039\right)$ reached significance. Thus, we computed separate two-way ANOVAs for each condition at electrodes P7 and P8 for the factors BLOCK (3) and LATERALITY (P7 vs. P8). For single letters, the interaction BLOCK x LATERALITY turned out to be significant $\left(\mathrm{F}_{(2,40)}=3.59, \mathrm{p}=.037\right)$. Thus, for single letters, separate one-way ANOVAs for the factor BLOCK (3) were computed. 
For electrode P7 we obtained a significant main effect of $\operatorname{BLOCK}\left(\mathrm{F}_{(2,40)}=3.49, \mathrm{p}=.05\right.$; block 1: $-5.65 \mu \mathrm{V}$, block 2: $-4.1 \mu \mathrm{V}$, block $3:-2.87 \mu \mathrm{V}$ ). For electrode P8, the main effect of BLOCK was not significant $(\mathrm{p}=.361)$. For words, the two-way ANOVA yielded a significant main effect of LATERALITY, but a non-significant main effect of BLOCK ( $p=.671)$ and a nonsignificant interaction of both factors $(\mathrm{p}=.392)$. For faces, neither a main effect nor the interaction turned out to be significant in the two-way ANOVA (all p's >.13). To summarize, N170 amplitude at posterior-temporal electrodes shows slight habituation for single letters, but not for words or faces.

\#\#\#\#\# Figure 1 near here \#\#\#\#\#

\#\#\#\#\# Figure 2 near here \#\#\#\#\#

\#\#\#\#\# Figure 3 near here \#\#\#\#\#

No significant correlations between measures of reading speed and N170-amplitude habituation (measured as the difference between block 1 and block 2 at electrodes P7 and P8, respectively) were obtained for single letters, words, or faces in children as well as adults (all p's > .228).

\section{Discussion}

In the present study, we investigated adaptation of the N170-component evoked by letters, words and faces in adults and seven to nine- year old children. For adults, we observed a tendency for smaller N170-amplitudes for single letters and words, but not for faces, in blocks two and three of the experiment compared to the first block. Thus, N170 amplitude habituates after a few repetitions of the relevant stimulus. For children, we only observed smaller N170amplitudes for single letters in blocks two and three compared to block one. No adaptation of N170-amplitude was obtained for words or faces. The amplitude reduction of the N170 for children was only present at electrodes placed over the left hemisphere of the brain. N170 adaptation to single letters and words did not correlate with reading speed. 
N170 amplitude adapts to single letters and words in adults. This is in line with previous findings (5, 16-18). Interestingly, Korinth et al. (5) showed that N170 amplitude to single letters adapts in fast, but not slow readers. The present sample consists of adult fast readers as indicated by above-average performance on measures of reading speed. Importantly, our findings extend these earlier results by showing that N170 amplitude also adapts for single letters in children aged seven to nine years.

Korinth et al. (5) reported a reliable correlation of reading speed and the amount of N170 reduction with repeated stimulus presentation. Here, we did not observe such a correlation (neither for adults, nor for children). Please note that for adults, text reading tasks were used to assess reading speed whereas in the task used during EEG-recording, single letters or words were employed as stimulus material. The use of different tasks for eliciting the N170 and for measuring reading speed in adults might be responsible for the absence of a reliable correlation between reading speed and N170-adaptation. Additionally, we observed a reliable adaptation of N170 amplitude in children for single letters, but not for words. In light of the absence of a correlation between single word reading speed and N170-peak amplitude adaptation we propose that adaptation of the N170 might depend on the perceptual experience with a certain stimulus class (here: letters) rather than on reading fluency. Children might have more perceptual experience with the processing of common letters like /a/ compared to specific single words such as /Spiel/.

N170 amplitude for words, pseudowords as well as for single letters has repeatedly been reported to be slightly left-lateralized $(3,9,11,20,21)$. In contrast to these findings, we did not observe clear evidence for lateralization of N170 amplitude in the present study. According to the phonological mapping hypothesis put forward by Maurer et al. (24), relative N170 leftlateralization emerges whenever automatic links between orthography and phonology are activated during the perception and processing of word-like stimuli. In contrast to the investigations by Bentin et al. (3), Kim et al., (21) and Maurer et al. $(9,11)$, the task used in the present investigation (detection of simple target stimuli) most likely required less phonological processing and, thus, automatic mapping processes between orthography and phonology were employed to a lesser degree than in the cited previous work. This could explain the absence of left-lateralization of N170 amplitude for letters and words in the present study.

We included face stimuli in the present study as a non-linguistic control condition (a familiar stimulus class for both, children and adults). In line with our expectations, we did not observe adptation of the N170 component at posterior-temporal electrodes (P7/P8) for children 
or adults in the face-condition. Please note that our analysis of N170 amplitude adaptation was restricted to the posterior-temporal channels P7 and P8 whereas previous studies found adaptation of N170 amplitude for faces at left and right occipital electrodes (e.g. 29). Furthermore, (19) showed lower N170 adaptation for women compared to men. As our sample of adults comprised of more women than men (14 vs. 7), only a small adaptation of the N170 would be expected at occipital electrodes (that we did not analyze), if at all. Note that the adaptor-stimulus interval was shorter in $(29 ; 200 \mathrm{~ms})$ than in the present study $(600 \mathrm{~ms}$ to $1200 \mathrm{~ms}$ ). Importantly, with the same stimulus parameters for linguistic and non-linguistic stimuli (i.e. ISI) we did not observe any N170 amplitude adaptation for the non-linguistic face stimuli for children and adults confirming that the observed adaptation effects at posteriortemporal channels are specific for linguistic material (letters, words).

Most previous research on adaptation to repeated presentation of faces compared two conditions: face followed by face vs. non-face followed by face. A decrease of ERP-amplitude to the second stimulus in these stimulus pairs in the face - face condition compared to the nonface - face- condition is taken as an index of habituation to the face stimulus (e.g. 16; please note that typically, the face- condition is realized in two different ways: same face - face and different face - face.). In the present study, we followed the approach of Korinth et al. (5) and Cao et al. (18) who presented the same stimulus in a block-wise arrangement. This difference in methodology could explain the absence of the habituation effect for faces.

\section{Conclusion}

We have shown for the first time that the amplitude of the N170-component of the event-related brain potential adapts to the repeated presentation of single letters in eight-year old children. Furthermore, in adults, N170 amplitude adapts to the repeated presentation of single letters as well as words. However, N170 adaptation did not correlate with measures of reading speed. We conclude that N170 adaptation might not be as strongly dependent on reading proficiency as previously thought; rather, it may be dependent on perceptual fluency with a specific stimulus class.

\section{Methods}

Participants. 21 neurotypical adults (14 women, 2 bimanual, all others right-handed, mean age: 25.08 yrs. range:18-35 yrs.) participated in the study. All were students at the 
University of Bamberg, native speakers of German and had normal or corrected to normal vision. Subjects were paid $10 €$ per hour or received course credit for their participation. Furthermore, 21 children ( 9 girls, age range 7 to 9 years, $M=8.4$ years) participated. All children were right handed and did not suffer from neurological or psychiatric diseases. They were recruited in primary schools in or close to Bamberg, were native speakers of German and had normal or corrected to normal vision. Children received two cinema vouchers each.

Ethics approval and consent to participate. The study protocol was conducted in accordance with the declaration of Helsinki and approved by the ethics committee of Bamberg University (Dossier number 2020-03/08). All participants gave their written informed consent prior to experimentation and were told that they could refrain from the study anytime without explanation. For children, the parents signed the consent form and were present throughout data acquisition. Children could refrain from the study at any time without explanation.

Design and procedure. First, reading abilities were assessed. The Saarbruecker Lesetexte consist of six easy texts with 180 words each (30). The adult participants were instructed to read each text as fast and as accurate as possible aloud to the instructor. Reading time needed for each story and the number of errors were recorded. Furthermore, the sum of the reading time and average reading time were computed. The Salzburger Lesetest (Hutzler \& Wimmer, personal communication) consists of 77 easy declaration sentences. Adult participants have to judge each sentence as true or false. Thus, the test assesses reading comprehension and reading speed. The number of correctly read sentences in three minutes is recorded.

Reading abilities of children were assessed with the one-Minute-Reading-Fluency subtest of the "Salzburger Lese- und Rechtschreibtest (SLRT II)". Children were requested to read aloud as many words or pseudowords as possible in one minute. The amount of words was noted by the experimenter.

The experiment consists of three conditions: letters, words, and faces. All stimuli were presented in white on a black background. Stimuli subtended $3 \mathrm{~cm}$ x $5 \mathrm{~cm}$ (words; $2.45^{\circ}$ x $4.09^{\circ}$ visual angle), $1 \mathrm{~cm} \times 1 \mathrm{~cm}$ (letters; $0.82^{\circ}$ ) or $7 \mathrm{~cm} \times 5 \mathrm{~cm}$ (faces; $5.73^{\circ} \times 4.09^{\circ}$ ) and were presented in the center of a computer monitor at a constant viewing distance of $70 \mathrm{~cm}$.

Similar to Korinth et al. (5), we used an oddball design with one standard and one target stimulus for each category. Stimuli of each category (single letters, words, faces) were 
presented in separate blocks (termed "conditions"). The stimulus material consisted of the letters "a" (target) and "c" (standard), the words "Spiel" (standard) and "Preis" (target). Two color photographs of a face of a female actor not known to the participants with a neutral emotional expression served as face stimuli (see Figure 4 for a depiction of the trial structure).

In each condition, 120 stimuli were presented for $700 \mathrm{~ms}$ without a break or intervening stimuli (80\% standards, $20 \%$ targets). The ISI varied randomly between $600 \mathrm{~ms}$ and $1200 \mathrm{~ms}$. Each condition was split into three thirds after recording for the analysis (Block A, B \& C) in order to observe possible attenuations of the N170 component over time (adaptation). The division into three blocks was not apparent for the participants. In each block 32 standards and 8 targets were presented in pseudorandom order (i.e. at least three standards had to occur after a target stimulus). Participants were instructed to press a button as fast as possible whenever a target stimulus was shown. The standard stimuli were not linked to a response. The three conditions were presented in randomized order (different for each participant).

\#\#\#\#\# Figure 4 near here \#\#\#\#\#

$50 \%$ of the participants had to respond with the right index finger, 50\% used the left index finger. A Cedrus RB 830 response box (www.cedrus.com) served to collect behavioral data. Presentation 17.0 software (neurobs.com) was used to deliver the stimuli and for recording the responses.

EEG-recording and data analysis. ERPs were recorded from 28 electrode sites mounted in an elastic cap (Easycap $\mathrm{GmbH}$, Herrsching, Germany) by means of sintered $\mathrm{Ag} / \mathrm{AgCl}$ electodes. The following sites were used: FP1, FP2, F7, F3, FZ F4, F8, FC5, FCZ, FC6, T7, C3, CZ, C4, T8, Cp5, CP1, CP2, CP6, P7, P3, PZ, P4, P8, O1, O2, TP9, TP10. Electrodes were placed according to the standard 10-20-system (31). For children, electrodes TP9/10 were not recorded. Otherwise, the recording procedure was identical for children and adults.

The ground electrode was placed on AFZ and the reference on the nose. Horizontal eye movements were recorded with two extra electrodes mounted at the right and left external canthus. Eye blinks were recorded with two electrodes placed above and below the left eye. All electrode impedances were maintained at or below $10 \mathrm{k} \Omega$. The electrophysiological signals 
were amplified with a bandpass ranging from 0.1 to $30 \mathrm{~Hz}$, digitized online with a $4 \mathrm{~ms}$ resolution (sampling rate of $250 \mathrm{~Hz}$ ) and stored on hard-disk for offline- analysis. A 32-channel brainamp DC amplifier (www.brainproducts.com) was used for EEG registration.

From the continuous EEG-signal, stimulus-locked epochs of $800 \mathrm{~ms}$ lengths starting $100 \mathrm{~ms}$ prior to stimulus onset were averaged for each condition and subject. Grand averages were formed separately for each condition and group. All stimuli requiring a response and standard trials with erroneous responses were discarded from further analysis. Thus, only standard stimuli without an (erroneous) response were analyzed. A pre-stimulus baseline of 100ms was used for ERP computation. Ocular artifacts were corrected using the ICA-procedure as implemented in BrainVisionAnalyzer-software (www.brainproducts.com; 32). In a second step, all epochs with amplitudes exceeding + or $-80 \mu \mathrm{V}$ at any electrode were rejected from further analysis. After artifact rejection, at least 20 trials (out of 32 per block in each condition) remained in the data for all children and adults.

ERP peak amplitude and latency were determined at electrodes P7 and P8. These electrodes showed the largest N170 amplitude for letters and words. A time-window of 100ms to $300 \mathrm{~ms}$ was used for N170 analysis. These data were submitted to a four-way, mixed design ANOVA with the between subjects' factor GROUP (2; adults vs. children) and the within subjects' factors CONDITION (3 levels; letters, words, faces), BLOCK (3 levels) and LATERALITY (left[P7] vs. right[P8]). Where appropriate, the degrees of freedom in the ANOVAs were adjusted to control for violations of the sphericity assumption (Huynh-Feldt correction). Degrees of freedom are reported before, p-values after the correction.

\section{References}

[1] Li, C, Ma, X, Zhu, C, Cao, X (2019). The recovery speed of category-sensitive N170 responses to faces and Chinese characters. Brain Research, 1723:146384. Doi: 10.1016/j.brainres.2019.146384

[2] Zhao, W, Chen, L, Zhou, C, Luo, W (2018). Neural correlates of emotion processing in a word detection task. Frontiers in Psychology, 9:832. doi: 10.3389/fpsyg.2018.00832 
[3] Bentin S, Mouchetant-Rostaing Y, Giard MH, Echallier JE, Pernier J (1999). ERP manifestations of processing printed words at different psycholinguistic levels: time course and scalp distribution. J Cogn Neurosci, 11,235-260.

[4] Maurer U, McCandliss B (2007). The development of visual expertise for words: the contribution of electrophysiology. In: E Grigorenko, A Naples (Eds.), Single word reading: Biological and Behavioral perspectives. 43-64. Mahwah, NJ: Lawrence Erlbaum Associates.

[5] Korinth SP, Sommer W, Breznitz Z (2013). Neuronal response specificity as a marker of reading proficiency: two-fold nature of the N170 revealed after massive repetition. Neuroreport, 24, 96-100.

[6] Stevens, C, McIlraith, A, Rusk, N, Niermeyer, M, Waller, H (2013). Relative lateralitiy of the N170 to single letter stimuli is predicted by a concurrent neural index of implicit processing of letter names. Neuropsychologia, 51, 667-674.

[7] Brem S, Lang-Dullenkopf A, Maurer U, Halder P, Bucher K, Brandeis D (2005). Neurophysiological signs of rapidly emerging visual expertise for symbol strings. Neuroreport, $16,45-48$.

[8] Emmorey, K, Midgley, KJ, Kohen, CB, Sehyr, ZS, Holcomb, PJ (2017). The N170 ERP component differs in laterality, distribution, and association with continuous reading measures for deaf and hearing readers. Neuropsychologia, 106, 298-309.

[9] Maurer, U, Rossion, B, McCandliss, B (2008). Category specificity in early perception: face and word N170 responses differ in both lateralization and habituation properties. Front Hum Neurosci, 2, 1-7.

[10] Maurer U, Schulz E, Brem S, van der Mark SV, Bucher K, Martin E, Brandeis D (2011). The development of print tuning in children with dyslexia: Evidence from longitudinal ERP data supported by fMRI. Neuroimage, 57, 714-722.

[11] Maurer U, Brem S, Bucher K, Brandeis D (2005). Emerging neurophysiological specialization for letter strings. J Cogn Neurosci, 17, 1532-1552.

[12] Maurer U, Brem S, Kranz U, Bucher K, Benz R, Halder P, Steinhausen H, Brandeis D (2006). Coarse neural tuning for print peaks when children learn to read. Neuroimage, 33, 749758. 
[13] Maurer U, Zevin B, McCandliss B (2008). Left-lateralized N170 effects of visual expertise in reading: Evidence from Japanese syllabic and logographic scripts. J Cogn Neurosci, 20,18781891.

[14] Boltzmann M, Rüsseler J (2013). Training-related changes in early visual processing of functionally illiterate adults: evidence from event-related brain potentials. BMC Neurosci, 14, 154.

[15] Grill-Spector, K, Henson, R, Martin, A (2006). Repetition and the brain: neural models of stimulus-specific events. Trends Cogn Sci, 10, 14-23.

[16] Cao, X, Ma, X, Qi, C (2015). N170 adaptation effect for repeated faces and words. Neuroscience, 294, 21-28.

[17] Fu, S, Feng, C, Guo, S, Luo, Y, Parasuraman, R (2012). Neural adaptation provides evidence for categorical differences in processing of faces and Chinese characters: an ERP study of the N170. PLoS One, 7:7

[18] Cao, X, Jiang, B, Gaspar, C, Li, C (2014). The overlap of neural selectivity between faces and words: evidences from the N170 adaptation effect. Exp Brain Res, 3015-3021.

[19] Mercure, E, Kadosh, KC, Johnson MH (2011). The N170 shows differential repetition effects for faces, objects, and orthographic stimuli. Front Hum Neurosci, 5, 1-10.

[20] Applebaum L, Liotti M, Perez R, Fox S, Woldoff M (2009) The temporal dynamics of implicit processing of non-letter, letter, and word forms in the human visual cortex. Front Hum Neurosci, 3,1-11.

[21] Kim K, Yoon H, Park H (2004). Spatiotemporal brain activation pattern during word/picture perception by native Koreans. Cogn Neurosci Neuropsychol, 15, 1099-1103.

[22] Tarkiainen A, Helenius P, Hansen P, Cornelissen P, Salmelin R (1999). Dynamics of letter sting perception in the human occipitotemporal cortex. Brain, 122, 2119-2131.

[23] Simos P, Breier J, Fletcher J, Foorman B, Mouzaki A, Papanicolaou A (2001). Age-related changes in regional brain activation during phonological decoding and printed word recognition. Dev Neuropsychol, 19, 191-210. 
[24] Maurer U, Blau VC, Yoncheva YN, McCandliss BD (2010). Development of visual expertise for reading: rapid emergence of visual familiarity for an artificial script. Dev Neuropsychol, 35, 404-422.

[25] Yoncheva YN, Blau VC, Maurer U, McCandliss B (2010). Attentioal focus during learning impacts N170 ERP responses to an artificial script. Dev Neuropsychol, 35, 423-445.

[26] Park J, Chiang C, Brannon EM, Woldorff, MG (2014). Experience-dependent hemispheric specialization of letters and numbers is revealed in early visual processing. J Cogn Neurosci, 26, 2239-2249.

[27] Wong AC-N, Gauthier I, Woroch B, DeBuse C, Curran T (2005). An early electrophysiological response associated with expertise in letter perception. Cogn Affect Behav Neurosci, 5, 306-318.

[28] Pernet C, Basan S, Doyon B, Cardebat D, Démonet D. Démonet F, Celsis P (2003). Neural timing of visual implicit categorization. Cogn Brain Res, 17, 327-338.

[29] Zhu, C, Ma, X, Ji, L, Chen, S, Cao, X (2018). Sex differences in categorical adaptation for faces and Chinese characters during early perceptual processing. Front Hum Neurosci, 11:656, doi: 10.3389/fnhum.2017.00656

[30] Kerkhoff, G, Wimbauer, K, Reinhart, S (2012). Saarbrücker Lesetexte. Universität des Saarlandes. AE Klinische Neuropsychologie. Gebäude A1.3, 66123 Saarbrücken.

[31] Jasper, HA (1958). The ten twenty electrode system of the international federation. Electroencephalogr Clin Neurophysiol, 10, 371-375.

[32] Joyce CA, Gorodnitsky IF, Kutas M (2004). Automatic removal of eye movement and blink artifacts from EEG data using blind component separation. Psychophysiology, 41, 313325

\section{Figure Captions}

Figure 1 
ERPs for adults at electrodes P7 and P8 for single letters (left), words (center) and faces (right). Solid line: first block, dashed line: second block, dotted line: third block. The adaptation of N170 amplitude for single letters and words, but not for faces, is clearly present.

Figure 2

ERPs for children at electrodes P7 and P8 for single letters (left), words (center) and faces (right). Solid line: first block, dashed line: second block, dotted line: third block. The adaptation of N170 amplitude for single letters, but not for words or faces, is clearly present.

Figure 3

Topographic maps of N170 peak amplitude for letters, words and faces for adults (top row) and children (bottom row) in the first block. On the right, the topography of the adaptation effect for letters and words is shown (peak amplitude of the difference block 1 - block 2).

Figure 4

Trial structure for the letter condition. A fixation dot is presented for $600 \mathrm{~ms}$ to $1200 \mathrm{~ms}$ (randomly varied ISI). Then, the stimulus is presented for $700 \mathrm{~ms}$ followed by the fixation dot. In the figure, two consecutive standard stimuli are shown. For the words and faces conditions, trial structure is identical but different stimuli are presented.

\section{Declarations}

\section{Conflict of Interest}

The authors declare that they have no competing interests.

\section{Author Contributions}

JR designed the study. UK acquired the data. JR and UK analysed the data and wrote the paper. 


\section{Funding}

None.

\section{Acknowledgments}

We thank Veronika Dippold for help in data acquisition.

\section{Data and material Availability Statement}

All data and material is available by request from the corresponding author jascha.ruesseler@uni-bamberg.de.

\section{Ethics approval and consent to participate}

The study protocol was conducted in accordance with the declaration of Helsinki and approved by the ethics committee of Bamberg University (Dossier number 2020-03/08). All participants gave their written informed consent prior to experimentation and were told that they could refrain from the study anytime without explanation. For children, the parents signed the consent form and were present throughout data acquisition. Children could refrain from the study at any time without explanation. 
Figures

ADULTS
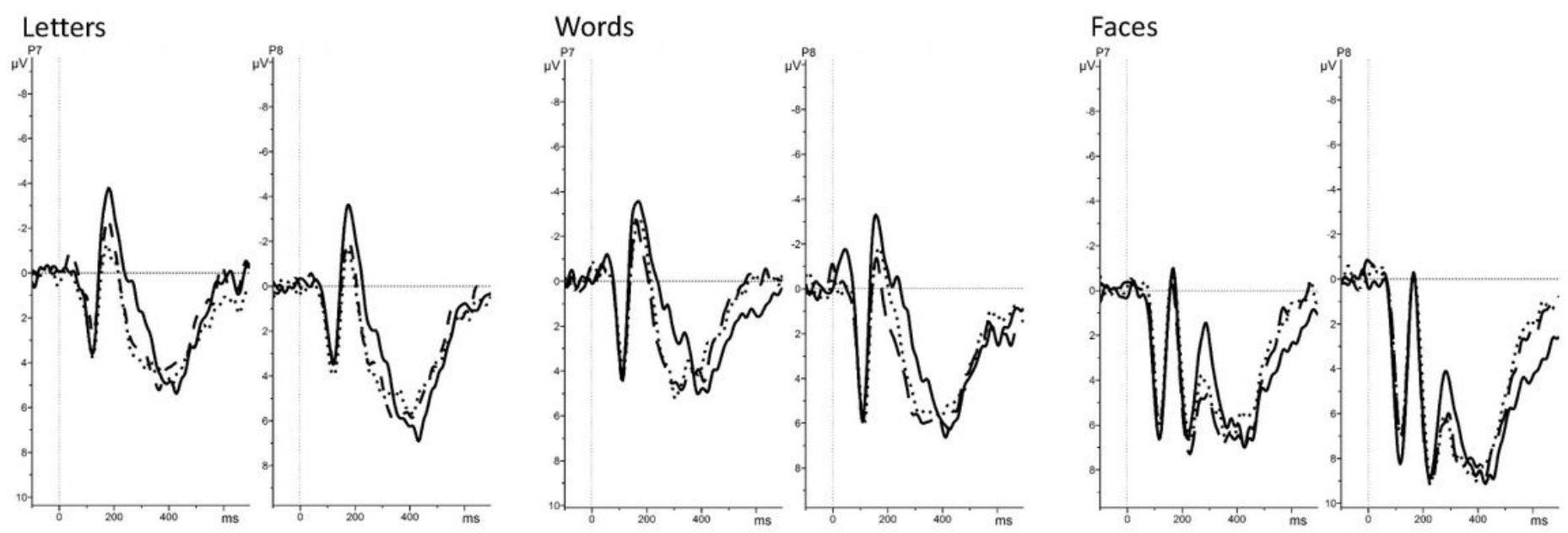

Block 1

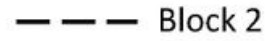

Block 3

\section{Figure 1}

ERPs for adults at electrodes P7 and P8 for single letters (left), words (center) and faces (right). Solid line: first block, dashed line: second block, dotted line: third block. The adaptation of N170 amplitude for single letters and words, but not for faces, is clearly present. 
CHILDREN

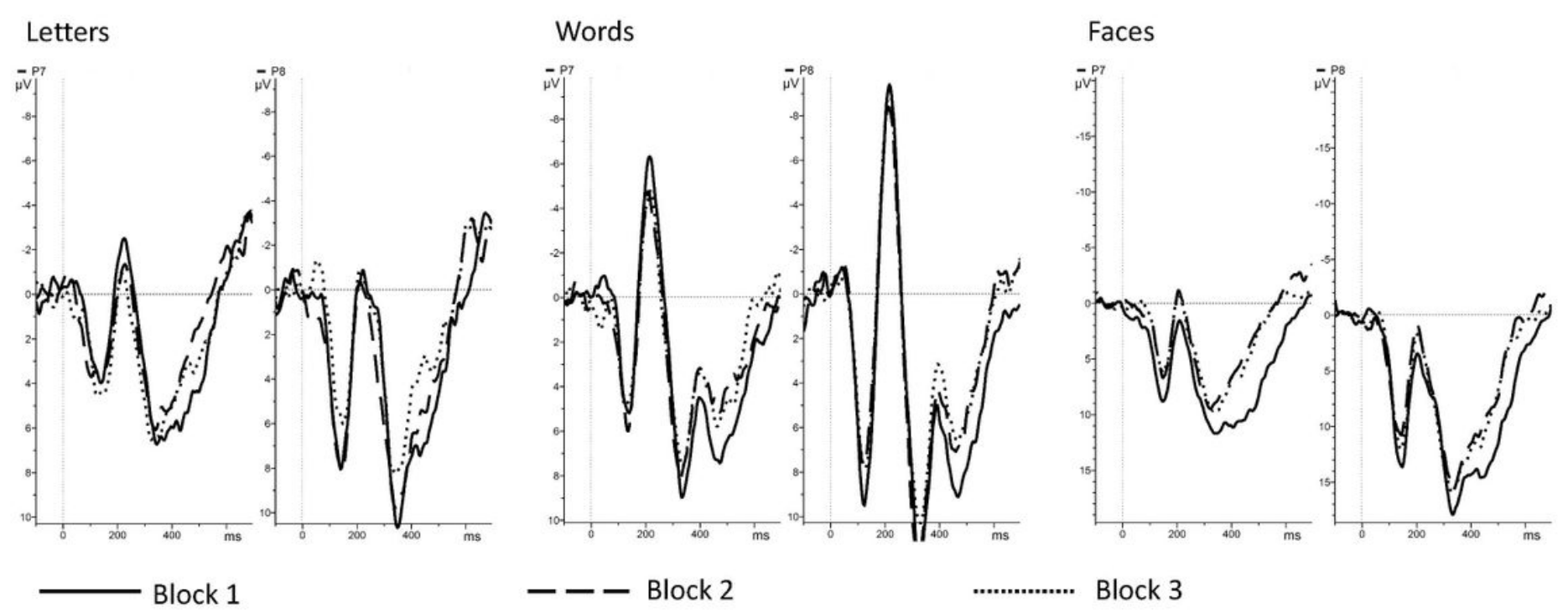

Figure 2

ERPs for children at electrodes P7 and P8 for single letters (left), words (center) and faces (right). Solid line: first block, dashed line: second block, dotted line: third block. The adaptation of N170 amplitude for single letters, but not for words or faces, is clearly present. 

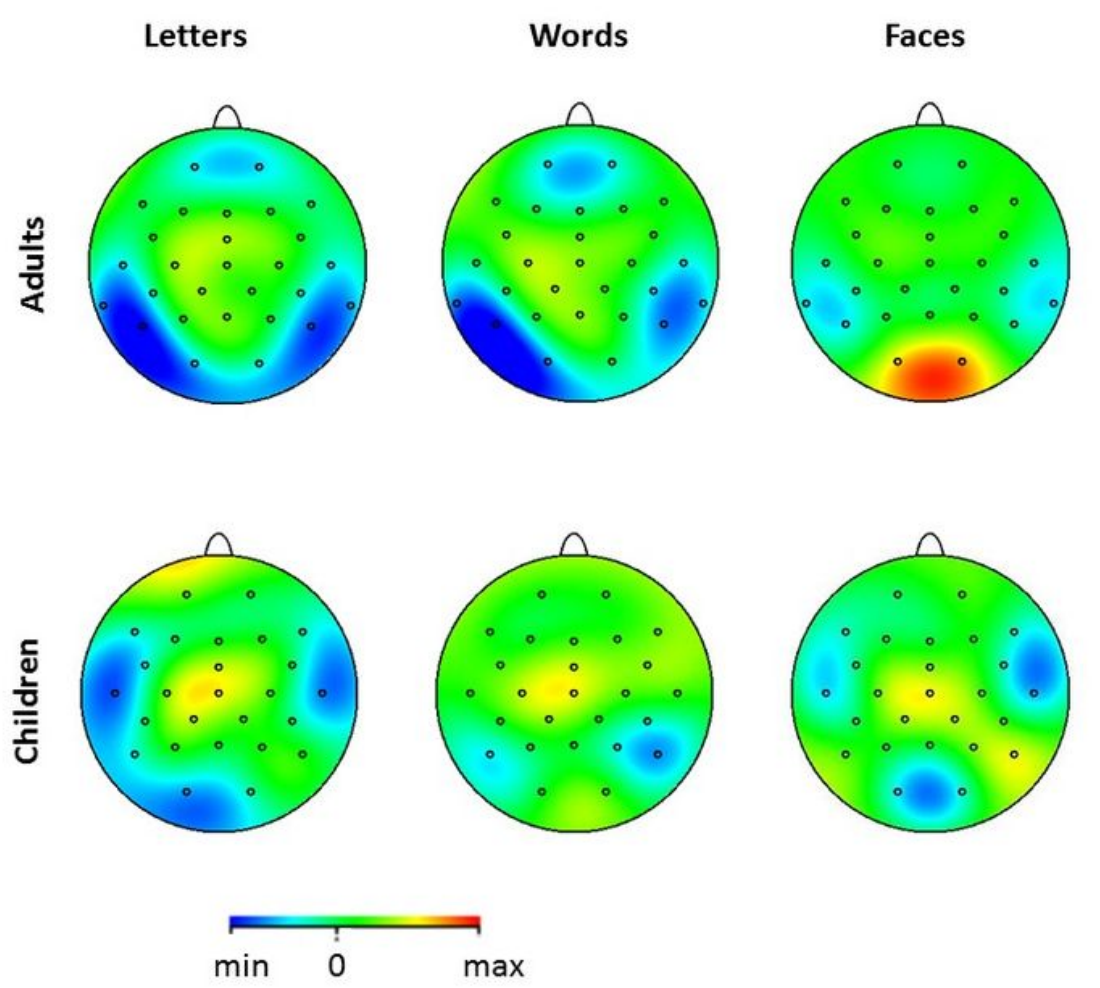

Letters Block 1-Block 2 Words Block 1-Block 2
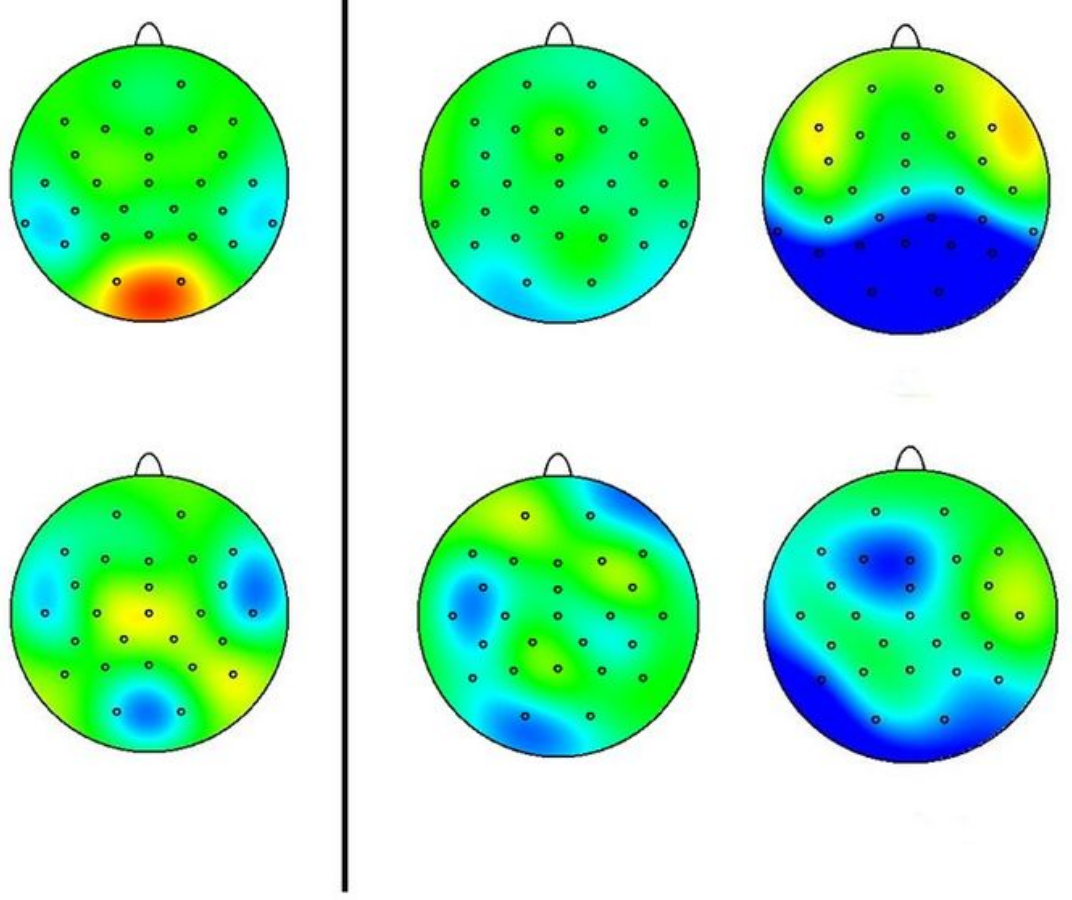

\section{Figure 3}

Topographic maps of N170 peak amplitude for letters, words and faces for adults (top row) and children (bottom row) in the first block. On the right, the topography of the adaptation effect for letters and words is shown (peak amplitude of the difference block 1 - block 2). 


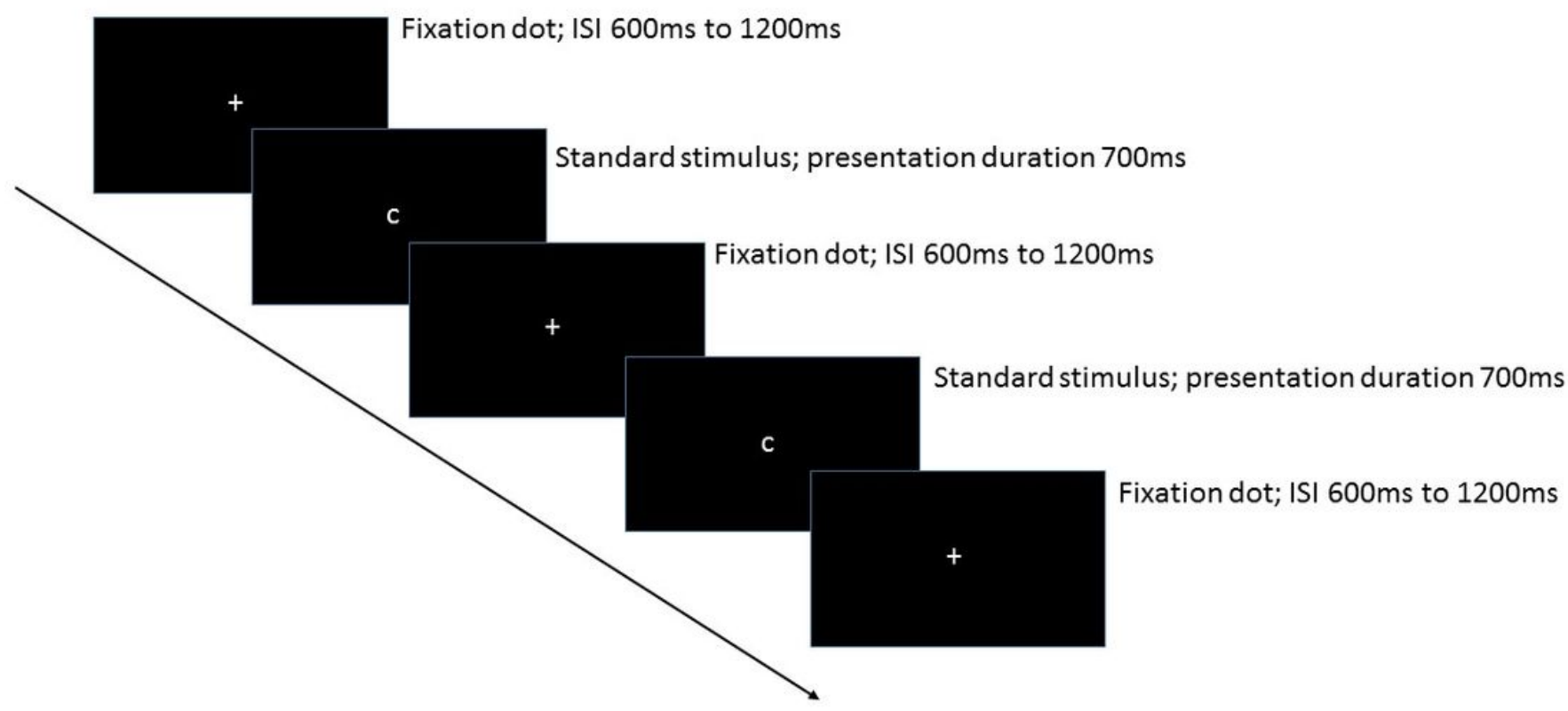

\section{Figure 4}

Trial structure for the letter condition. A fixation dot is presented for $600 \mathrm{~ms}$ to $1200 \mathrm{~ms}$ (randomly varied ISI). Then, the stimulus is presented for $700 \mathrm{~ms}$ followed by the fixation dot. In the figure, two consecutive standard stimuli are shown. For the words and faces conditions, trial structure is identical but different stimuli are presented. 